\title{
How do changes in speed affect the perception of duration?
}

William J. Matthews

University of Essex, UK

Running head: Speed changes and time perception

Keywords: time perception; internal clock; speed; acceleration; deceleration; temporal estimation; temporal reproduction

\section{Author note}

Affiliation: William J. Matthews, Department of Psychology, University of Essex.

Acknowledgements: This work was supported by a grant from the University of Essex Research Promotion Fund. I am grateful to Rebeccah Billing for helpful discussion and assistance with data collection. I thank Simon Grondin, Neil Stewart, Mair Thomas, John Wearden, and two anonymous reviewers for comments on earlier versions.

Correspondence address: William Matthews, Department of Psychology, University of Essex, Colchester, CO4 3SQ, United Kingdom, E-mail: will@essex.ac.uk 


\begin{abstract}
Six experiments investigated how changes in stimulus speed influence subjective duration.

Participants saw rotating or translating shapes in three conditions: constant speed, accelerating motion, and decelerating motion. The distance moved and average speed were the same in all three conditions. In temporal judgment tasks, the constant-speed objects seemed to last longer than the decelerating ones, which in turn seemed to last longer than the accelerating stimuli. In temporal reproduction tasks, the difference between accelerating and decelerating stimuli disappeared; furthermore, watching an accelerating shape lengthened the apparent duration of the subsequent (static) display. These results (a) suggest that temporal judgment and reproduction can dissociate for moving stimuli because the stimulus influences the apparent duration of the subsequent interval, and (b) constrain theories of time perception, including those which emphasize memory storage, those which emphasize the existence of a pacemaker-accumulator timing system, and those which emphasize the division of attention between temporal and non-temporal information processing.
\end{abstract}




\section{Time winding down: How do changes in speed affect the perception of duration?}

Suppose that you are standing on the platform of a railway station. Whilst waiting for your train you watch several others move past: One glides through the station at a steady pace; a second pulls out of the platform opposite and gathers speed before it disappears around the corner; and a third hurtles out of a tunnel and slows to a halt to pick up passengers. Assuming that all three trains are visible for the same length of time, will these three events all seem to have equal duration? Or will the nature of the movement affect your perception of time?

Several investigators have examined the effects of stimulus speed on time perception. The most widely-repeated finding is that moving stimuli seem to last longer than static ones (e.g., S. W. Brown, 1995; Kanai, Paffen, Hogendoorn, \& Verstraten, 2006; Lhamon \& Goldstone, 1975), and that apparent duration increases with increasing stimulus speed (e.g., Beckmann \& Young, 2009; S. W. Brown, 1995; Kaneko \& Murakami, 2009; Leiser, Stern, \& Meyer, 1991). Some caution is needed before accepting the generality of these conclusions because the results are context-dependent: a stationary oddball shown in a train of moving stimuli will have longer subjective duration (Tse, Intriligator, Rivest, \& Cavanagh, 2004).

The effects of stimulus speed on subjective duration have implications for any model of time perception. Theories of time perception can be grouped into two broad classes (Lejeune, 1998). The first class assumes that duration is a by-product of non-temporal information processing; there is no specialized "timer" for measuring duration. For example, Ornstein (1969) argued that perceived duration depends upon the space needed to store the stimulus in memory, and others have stressed that time perception depends on the number of changes taking place during an interval (e.g., Block \& Reed, 1978; Poynter, 1983). These storage- or change-based models have typically been applied to retrospective time judgments - where the participant does not know in advance that they will be asked for a time estimate - but they have also been related to the effects of stimulus motion on time perception in prospective tasks, where the observer knows that a time judgment will be required: More complex motion patterns are taken to lengthen apparent duration (Aubry, Guillaume, Mogicato, Bergeret, \& Celsis, 2008) and faster speeds are taken to entail greater positional change and, accordingly, longer subjective time (S. W. Brown, 1995; Poynter, 1989).

The second class of models assumes a specialized mechanism for measuring time, based upon some kind of accumulation or counting process (e.g., Gibbon, Church, \& Meck, 1984; Rammsayer \& Ulrich, 2001; Treisman, 1963; Treisman, Faulkner, Naish, \& Brogan, 1990). There is usually taken to be an internal pacemaker or oscillator which emits pulses that are accumulated until stimulus offset, at which point the number of counts in the accumulator provides the measure of 
duration. A subset of these pacemaker models include the idea that attention must be divided between temporal processing and non-temporal processing, such that directing attention away from the timer reduces pulse accumulation and shortens subjective duration (e.g., Zakay \& Block, 1997). Stimulus speed has been argued to affect the pacemaker, with faster speeds producing higher accumulation rates and longer subjective time (Kaneko \& Murakami, 2009).

The current work builds on previous investigations of the effects of movement on time perception by asking, for the first time: how do changes in speed affect the perception of duration? That is, if a moving stimulus remains visible for a fixed time, and travels a fixed distance, does its apparent duration depend on whether it accelerates, decelerates, or moves at a constant speed? Existing models do not generate clear predictions regarding the effect of changes to stimulus speed on subjective duration because the relationship between stimulus movement and the relevant theoretical constructs is rather under-specified. For example, does storage size depend purely on distance moved - in which case accelerating, decelerating, and constant-speed stimuli will have the same subjective duration - or do changes in speed entail an additional complexity, such that constant-speed stimuli seem shorter than accelerating and decelerating objects? Similarly, pacemaker-accumulator models do not provide strong predictions as they depend on the assumed relationship between stimulus speed and pacemaker rate.

The aim of the current experiments is therefore to establish the phenomenology of the effects of changes in speed on subjective duration. These effects will not allow us to choose between competing models of time perception, but they will provide an important constraint on how these theoretical positions accommodate the effects of stimulus motion on subjective time.

\section{Experiment 1}

Experiment 1 compared the apparent durations of rotating shapes which accelerated, decelerated, or moved with constant speed. Participants rated the apparent durations of moving shapes on a nine-point scale ranging from 1 (very short) to 9 (very long) (Goldstone \& Goldfarb, 1964). For a given physical duration, distance moved and mean speed were kept constant while the rate of change in speed varied.

Two points should be clarified from the outset. The first is that average speed, acceleration rate, and distance travelled are unavoidably confounded. If a stimulus moves with constant velocity $v$ for a length of time $t$, one can display an accelerating stimulus for the same duration and with the same mean velocity so that the distance travelled is the same. If the duration is then increased, one has to choose between keeping the mean velocity the same as before (in which case the rate of 
acceleration decreases and the distance travelled increases), keeping the rate of acceleration constant (in which case the distance travelled and mean velocity will increase), or changing the starting velocity. In the current experiments, mean speed was held constant across conditions as this seemed simplest. The intrinsic relationships between time, distance, and rate of acceleration mean that it is difficult to interpret any interaction between type of movement (accelerating or constant velocity) and stimulus duration.

The second point to note is that the present experiments are concerned with apparent duration, not with the accuracy of judgment. Studies of the accuracy of time judgments typically vary the duration of some comparison stimulus with respect to a standard and require a discrimination judgment (e.g., Ulrich, Nitschke, \& Rammsayer, 2006b). Such an approach will be difficult here because varying the comparison duration will necessarily alter some other aspect of the stimulus. The current emphasis is therefore upon how stimulus dynamics affect perceived duration.

\section{Method}

Participants. Twenty four participants took part ( 8 male; mean age 20.4 years, $S D=4.9$ years). Most received course credit; one volunteered.

Stimuli. The stimuli were six 2D shapes (two rectangles, two squares, two crosses of varying colours). The shapes were presented against a white background and measured no more than $256 \mathrm{x}$ 256 pixels (sizes ranged from approximately $1.5 \times 1.7$ degrees to $3.5 \times 4.0$ degrees visual angle). Stimuli were displayed on a 19" CRT monitor ( $1280 \times 1024$ pixels refreshing at $85 \mathrm{~Hz})$, viewed from approximately $1 \mathrm{~m}$ through the glass window of a sound-attenuating chamber. Stimulus timing was controlled in terms of screen refreshes using PsychoPy (Peirce, 2007). The shapes rotated around their centre about an axis perpendicular to the screen. In the constant speed condition, rotation was a fixed 225 degrees per second (3 degrees per refresh). In the accelerating condition, the shape smoothly accelerated from stationary such that the total distance moved (and hence the mean speed) was the same as in the constant speed condition. The motion dynamics of the decelerating condition mirrored those of the accelerating condition: the shape smoothly slowed from the maximum speed of the accelerating condition to stationary. Stimuli were presented for either $2 \mathrm{~s}$ or $3 \mathrm{~s}$. The starting orientation of each shape was a randomly selected integer from 0-359 degrees.

Design and Procedure. The study employed a 2 (duration, 2 s vs. 3 s) by 2 (movement direction, clockwise vs. anticlockwise) by 3 (motion: constant, accelerating, decelerating) withinsubjects design. Participants judged each shape once in each combination of conditions, giving 72 
test trials. Trial order was randomized for each participant. Participants completed 10 practice trials during which stimuli (shape, movement, duration, direction) were randomly selected. The sequence of events on each trial was: a blank interval for 706-1412 ms, a blank fixation cross for $353 \mathrm{~ms}$, a blank interval for $529-882 \mathrm{~ms}$, followed by the stimulus (the slightly odd timings result from a minor coding error). After stimulus offset, participants rated its apparent duration on a scale ranging from 1 (very short) to 9 (very long). In all experiments, participants were instructed not to count or use a watch to time the stimulus but to rely on their subjective impressions. Across all experiments, a small number of trials were excluded from analysis because of timing errors (dropped frames) during stimulus display.

\section{Results and Discussion}

The results are shown in Figure 1. An initial analysis indicated no effect of rotation direction, which was therefore dropped from the analysis. A 2 (duration) $\times 3$ (condition) repeated measures ANVOA indicated that, as one would expect, $3 \mathrm{~s}$ stimuli were judged to last longer than $2 \mathrm{~s}$ ones, $F(1$, $23)=126.6, p<.001, \eta_{p}^{2}=.85$. More importantly, motion dynamics affects rated duration, $F(2,46)=$ 45.6, $p<.001, \eta_{p}^{2}=.67$; constant-speed shapes seemed to last longer than decelerating objects, which in turn seemed to last longer than accelerating stimuli. All Bonferroni-corrected pairwise comparisons were significant (all corrected $p s<.007$ ). Motion condition also interacted with duration, $F(2,46)=11.72, p<.001, \eta_{p}^{2}=.34$. The interaction reflects the fact that the effect of stimulus duration ( $2 \mathrm{~s}$ vs. $3 \mathrm{~s}$ ) is greater for the constant velocity condition than for the accelerating and decelerating conditions. This effect did not replicate in the subsequent experiments and, as noted above, the interaction is difficult to interpret because the final/starting speeds in the accelerating and decelerating conditions vary with the duration in order to keep the mean speed constant. It is worth noting that, because mean speed was held constant across conditions, the distance travelled was greater in the $3 \mathrm{~s}$ condition than in the $2 \mathrm{~s}$ condition, and participants may have used this distance cue as part of the basis for their temporal judgments. However, for both physical durations, the distances travelled and mean velocities were the same for the accelerating, decelerating, and constant speed conditions; only the change in stimulus speed can underlie the observed differences in perceived duration. (The same is true of the other experiments reported below.)

Experiment 1 shows that changes in stimulus speed influence perceived duration, and produced the surprising result that constant speed stimuli seem to last longer than either accelerating or decelerating shapes. Experiment 2 sought to clarify two methodological points. 
Firstly, Experiment 1 used a subjective rating scale which the participant can use as he or she wishes. Additional research from my lab suggests that judgments using this kind of scale are no different from when the categories correspond to specific objective durations and the task is to identify the stimuli as accurately as possible (e.g., response $1=0.75 \mathrm{~s}$ ), but it is worth checking that the results are not due to the use of this category judgment task. Secondly, perceptual judgments of a given stimulus are influenced by the other items present in the experiment. For example, Gomez and Robertson (1979) found that the oft-repeated positive relationship between stimulus size and apparent duration only holds when both small and large items are intermixed in an experimental session. Experiment 1 used a within-subjects design; perhaps the accelerating and decelerating shapes are more perceptually similar, such that the constant speed condition "stands out" and correspondingly seems to have longer duration (Pariyadath \& Eagleman, 2007; Tse et al., 2004; Ulrich et al., 2006b). Experiment 2 used a between-subjects design and participants made absolute judgments of duration on a millisecond scale.

\section{Experiment 2}

\section{Method}

Participants. A total of 60 participants were recruited (the number suggested by an a priori power calculation) but technical problems meant that only 51 provided data (11 males, mean age 21.4 years, $S D=6.2$ years; 11 had completed the previous experiment but were naive as to the results). Participants received course credit or $€ 3$.

Stimuli. The stimuli and apparatus were similar to those in Experiment 1. Four shapes were used ( $a$ filled square, an unfilled square, a rectangle, a cross,) and all were circumscribed to be no greater than $256 \times 256$ pixels ( $1.7 \times 1.9$ to $3.5 \times 4.0$ degrees). In the constant velocity condition, rotation was a fixed 2 degrees per refresh (170 degrees per second). In the accelerating condition the shape smoothly accelerated from stationary such that the total distance moved (and hence the mean velocity) was the same as in the constant velocity condition. In the decelerating condition the shape smoothly slowed from the maximum velocity of the accelerating condition to stationary. Stimuli were presented for five different durations: 1000, 1247, 1494, 1741, 1988 ms (respectively equal to $85,106,127,148$, or 169 refreshes). The starting orientation of each shape was a randomly selected integer from 0-359 degrees.

Design and Procedure. Seventeen participants were assigned to each of the constant speed, accelerating, and decelerating conditions. Each participant completed forty test trials, one presentation of each shape moving in each direction (clockwise/anticlockwise) for each of the 5 
durations. Participants completed 4 practice trials during which stimuli (shape, movement, duration, direction) were randomly selected for judgment.

The sequence of events on each trial was: blank interval for 1000-2000 ms, fixation cross for $500 \mathrm{~ms}$, blank interval for $750-1250 \mathrm{~ms}$, stimulus presentation for $1000-1988 \mathrm{~ms}$, followed by a blank interval during which the participant typed his or her judgment of the duration of the stimulus. Participants typed their judgments in milliseconds. (They were reminded that $1000 \mathrm{~ms}=1 \mathrm{~s}$, so that, for example, 1.25 seconds $=1250 \mathrm{~ms}$ ). They were told that no stimulus would be shorter than 100 ms or longer than $3000 \mathrm{~ms}$ and that they should be as precise as they could but that they need not agonize over every decision. Responses outside the range 100-3000 ms were excluded. Participants could see the values they typed appear on the screen, and could clear the entries and retype their judgments before pressing the Return key to register the response and progress to the next trial. $\mathrm{A}$ handful of trials were excluded because of missing responses.

\section{Results and Discussion}

The results are shown on the left of Figure 2. Inspection suggests that longer-lasting stimuli are judged to last longer, and judgments are affected by motion: constant speed stimuli have the longest perceived duration, followed by decelerating shapes, with accelerating objects having the briefest subjective duration. A 3 (condition: constant, accelerating, decelerating) $\times 5$ (stimulus duration) mixed ANOVA revealed, confirmed these impressions, with a main effect of duration, $F(3.32,159.37)=260.3, p<.001, \eta_{p}^{2}=.84$ (here and at certain points below, a Huynh-Feldt correction was applied because of violations of sphericity), a main effect of condition, $F(2,48)=3.28$, $p=.046, \eta_{p}^{2}=.12$, but no interaction, $F(6.64,159.37)=1.67, p=.123, \eta_{p}^{2}=.07$. Bonferroni-corrected pairwise comparisons indicating that the constant velocity and accelerating conditions differed $(p=$ .041); no other comparisons were statistically significant ( $p s>.5$ ), but this is likely due to the low power of a between-subjects experiment with only 17 participants per condition.

As an additional analysis, I examined the variability of magnitude estimates. The coefficients of variation (standard deviation of judgment divided by mean judgment) are shown in the right panel of Figure 2. Variability decreased as the duration increased, $F(3.41,163.55)=7.54, p<.001$, $\eta_{p}^{2}=.14$, but neither movement nor the movement-duration interaction were significant, $F_{\mathrm{s}}<1$. The variability of temporal judgments is important to models of timing (e.g., Gibbon et al., 1984; Ulrich, Nitschke, \& Rammsayer, 2006a) but the interpretation of variability results from verbal estimation tasks is not always straightforward (see e.g., Wearden, Norton, Martin, \& Montford- 
Bebb, 2007). The coefficient of variation data are therefore included for completeness, but will not be discussed further.

To summarize: Experiment 2 therefore replicates the results of Experiment 1. Shapes rotating with constant speed seem to have longer duration than those which decelerate, which in turn seem to have longer duration than those which accelerate. This is true even when observers only see one type of movement in the course of the experiment, and when they are attempting to estimate the objective duration of the stimulus as accurately as they can.

Experiments 1 and 2 used rotational motion, which may have a privileged status in time perception because of its periodicity. Experiment 3a sought to replicate the foregoing pattern of results using translational motion.

\section{Experiment 3a}

\section{Method}

Participants. Twenty students from the University of Essex participated in exchange for course credit or $f 3$ (18 female, mean age $19.1, S D=1.0$ ). Nine had participated in one or both of the experiments using rotating stimuli.

Stimuli. The stimulus was a black square ( $128 \times 128$ pixels, approximately 1.9 degrees visual angle) presented against a white background. In the constant velocity condition, the square moved at a constant 5 pixels per refresh (approximately 6.6 degrees visual angle per second). The accelerating and decelerating conditions were like those in Experiments 1 and 2 and involved smooth changes in speed with the same mean speed as the constant velocity case. Stimuli were presented for $588,941,1294$, or $1647 \mathrm{~ms}(50,80,110$, or 140 frames). The square was always presented halfway up the screen; its horizontal starting position was randomly chosen on each trial such that the movement never carried the centre of the square outside an invisible boundary 400 pixels either side of the centre of the screen.

Design and Procedure. The experiment employed a 3 (movement: constant velocity, accelerating, decelerating) $\times 4$ (stimulus duration: 588, 941, 1294, $1647 \mathrm{~ms}$ ) within subject design. Participants were told they would receive 10 practice trials (although a programming error meant that 8 participants only received 1 practice trial), during which stimuli (shape, movement, duration, direction) were randomly selected for judgment. They then completed 96 test trials, comprising 8 presentations of each of the 4 stimulus durations in each of the 3 conditions. Movement was left to right for half of the trials in each condition and right to left in the other half. Participants were invited to take a short break after 32 and 64 test trials. 
The sequence of events on each trial was as Experiment 2. After stimulus offset the participant rated the apparent duration of each stimulus on a 9-point scale anchored at 1 (very short) and 9 (very long) in the same way as Experiment 1.

\section{Results and Discussion}

The results are shown in the left panel of Figure 3. A $2 \times 3$ within-subjects ANOVA indicated that longer physical durations were rated as lasting longer, $F(1.90,36.13)=369.03, p<.001, \eta_{p}^{2}=$ .95 , and that constant velocity stimuli were perceived as lasting longer than decelerating stimuli, which were perceived as lasting longer than accelerating stimuli, $F(2,38)=23.63, p<.001, \eta_{p}^{2}=.554$. Post-hoc tests revealed that all movement conditions differed from one another (all Bonferronicorrected $p s<.02)$. Duration and movement condition did not interact, $F(6,114)=2.15, p=.053, \eta_{p}^{2}$ $=.10$.

For translation motion, as for rotation, the apparent duration follows the order constant speed > decelerating > accelerating. So far, all of the experiments have assessed participants' judgments of the stimulus duration. An alternative approach is to require participants to reproduce the duration of the moving stimulus (e.g., S. W. Brown, 1995; Kanai et al., 2006). Experiment 3b replicated Experiment 3a but used a reproduction paradigm to assess apparent duration.

\section{Experiment 3b}

\section{Method}

Participants. Twenty five students from the University of Essex took part (20 female; mean age 19.3 years, $S D=2.0$ years); some received course credit, others were paid $£ 4$. None had participated in Experiment 3a; 9 had completed one or both of the earlier experiments.

Stimuli, Design, and Procedure. The stimuli and design were identical to Experiment 3a, except that after stimulus offset there was a blank interval during which the participant reproduced the stimulus duration by holding down the left mouse button. Participants were instructed to wait until the stimulus had disappeared before responding (they could respond at any time after stimulus offset). Release of the mouse button triggered the next trial.

\section{Results}

Responses shorter than 100 ms or longer than 4000 ms were discarded as mistakes; trials on which the latency from stimulus offset to response was less than $150 \mathrm{~ms}$ were also discarded as 
anticipatory responses. (Across the whole experiment, fewer than $2.7 \%$ of trials were discarded because of display errors, extreme or anticipatory responses.)

The results are shown in the centre of Figure 3 . As one would expect, reproduced duration increased with stimulus duration, $F(1.61,38.60)=240.37, p<.001, \eta_{p}^{2}=.91$. There was also a main effect of movement condition, $F(2,48)=26.98, p<.001, \eta_{p}^{2}=.53$, but no interaction, $F(5.21,124.94)$ $=1.88, p=.099, \eta_{p}^{2}=.07$. Bonferroni-corrected pairwise comparisons revealed that the constant velocity condition was significantly different from both the accelerating and decelerating conditions ( $p$ s $<.001)$ which did not differ $(p=.438)$.

Experiment $3 \mathrm{~b}$ replicated the finding that the constant velocity condition was perceived as lasting longer than the accelerating and decelerating conditions, but did not replicate the difference between these latter conditions. This is unlikely to have been due to statistical power: in Experiment $3 a$, the effect size (Cohen's $d$ ) for the difference between acceleration and deceleration was 0.74 , so that the power to detect this effect in the current experiment (even after the Bonferroni-adjusting the alpha levels to .017) was approximately 85\% (Erdfelder, Faul, \& Buchner, 1996). [This power calculation is based on the effect size observed in Experiment 3a (see e.g., Greenwald, Gonzalez, Harris, \& Guthrie, 1996), although some authors have queried this approach (e.g., Miller, 2009) so caution regarding the exact power estimate may be appropriate.]

This failure to replicate is surprising, and may be due to the fact that participants were free to begin their reproduction at any point after stimulus offset. If stimulus motion affects the time at which reproduction begins, this may in turn influence the reproduced duration as memory for the target duration is likely to change over time (Wackermann \& Ehm, 2006; Wearden \& Ferrara, 1993). In Experiment 3c, the beginning of the reproduction interval was controlled.

\section{Experiment 3c}

\section{Method}

Participants. Twenty students from the University of Essex took part (14 female; mean age 19.4 years, $S D=3.1$ years) for course credit or $£ 3$. Four had participated in one or both of Experiments 1 and 2; none had participated in Experiments 3a or 3b.

Stimuli, Design, and Procedure. The stimuli, design, and procedure were identical to Experiment $3 \mathrm{~b}$ except for a change in the way the stimulus duration was reproduced. Following stimulus offset, the screen was blank for $756 \mathrm{~ms}$ (65 frames) after which the black square reappeared in the centre of the display. Participants were instructed to tap the spacebar when the 
second, stationary square had been on the screen for the same length of time as the moving square. When the participant pressed the spacebar, the square disappeared and the next trial began.

\section{Results and Discussion}

As before, responses shorter than $100 \mathrm{~ms}$ or longer than $4000 \mathrm{~ms}$ were discarded as mistakes. The results are shown in the right panel of Figure 3. As one would expect, reproduced duration increased with stimulus duration, $F(2.11,40.17)=359.32, p<.001, \eta_{p}^{2}=.95$. There was also a main effect of movement condition, $F(2,38)=14.90, p<.001, \eta_{p}^{2}=.44$, but no interaction, $F(6,114)$ $=1.09, p=.374, \eta_{p}^{2}=.05$. Bonferroni-corrected pairwise comparisons revealed that the temporal reproductions from the constant speed condition were significantly longer than those from both the accelerating $(p<.001)$ and decelerating conditions $(p=.001)$ which did not differ $(p=1.000)$. The results therefore replicate those of Experiment $3 \mathrm{~b}$ under conditions in which the onset of the temporal reproduction is controlled. The power to detect a difference between the accelerating and decelerating conditions of the size found in Experiment 3a was approximately 75\%. The power to detect an effect in one or both of Experiments $3 b$ and $3 c$ is therefore $1-\left(0.15^{*} 0.25\right)=96 \%$, so the lack of an effect is unlikely to be due to low power.

One possible explanation for the difference between the results using judgment tasks and those using reproduction tasks is that the different types of stimulus motion - acceleration, deceleration, or constant speed - differentially affect the apparent duration of stimuli that come after the moving object. Temporal reproduction requires the second interval (the reproduction) to be matched to the first. If the passage of subjective time during that second interval depends upon the nature of the original stimulus, there may be a dissociation between the reproduced duration and the judgment of the original duration. Experiment 4 asks whether acceleration, deceleration, and constant speed differentially affect the perception of stimuli that come after the moving shape.

\section{Experiment 4}

This experiment was similar to Experiments 3a-3c. A square translated across the screen with either constant, accelerating, or decelerating velocity, and was then replaced by a static square. The moving square had the same duration on every trial, and was not the subject of judgment. The second square remained on-screen for a variable amount of time and participants were required to rate its duration on a nine-point scale. Because it is the static square which is to be judged, the conditions are referred to as post-constant, post-acceleration, and post-deceleration. 


\section{Method}

Participants. Twenty seven participants (19 female; mean age 21.5 years, SD $=8.0$ years) took part for course credit or $£ 3$. Some had participated in previous experiments.

Stimuli, Design, and Procedure. Participants were tested in quiet cubicles, viewing the screen from approximately $1 \mathrm{~m}$. On each trial, a blue square moved across the screen for $1647 \mathrm{~ms}$ (140 frames) with either constant, accelerating or decelerating velocity, exactly as in Experiments 3a3c. After a 756-ms (65 frame) blank interval, a static red square appeared for 588, 941, 1294, or 1647 ms $(50,80,110$, or 140 frames). Participants rated the duration of this red square on a nine-point scale, in the same way as for Experiments 1 and 3a. Participants were told that they should study the moving square while it was on the screen, but that they only needed to judge the static square.

\section{Results and Discussion}

The data are shown in the left panel of Figure 4; the right panel shows the results collapsed across duration. Longer-lasting stimuli were judged to last longer, $F(1.53,39.75)=270.03, p<.001$, $\eta_{p}^{2}=.91$. Judgments were also affected by movement condition, $F(2,52)=3.67, p=.032, \eta_{p}^{2}=.12$, but there was no interaction, $F<1$. Inspection of the means in the right panel of Figure 4 suggests that the post-accelerating condition produced smaller ratings than the post-decelerating and postconstant speed conditions, which did not differ much. Bonferroni-corrected pairwise comparisons partially confirmed this impression: the post-accelerating condition was judged shorter than the post-constant speed case, $p=.018$, but the difference between post-accelerating and postdecelerating conditions did not survive Bonferroni correction $(p=.101$; the uncorrected $p$ value is significant). As one would expect based on Figure 4, the post-constant speed and post-decelerating conditions did not differ $(p=1.00)$. The finding that stimulus motion affects the apparent duration of the subsequent static display provides an explanation for the dissociation between time judgment and time reproduction seen in Experiments 3a, 3b and 3c; this idea is discussed in detail below.

\section{General Discussion}

The results may be summarized as follows. Experiment 1 found that rotating shapes are perceived to last longer when they move with constant speed than when they decelerate, which in turn seem to last longer than when they accelerate. Experiment 2 showed that this was not due to intra-experiment stimulus comparisons or the choice of judgment task: trying to estimate physical 
duration in milliseconds when one has only encountered one type of motion in the experiment produces the same pattern. The results generalize to translational motion (Experiment 3a) but when a temporal reproduction task is used the difference between acceleration and deceleration disappears - although both still seem shorter than constant speed motion (Experiments $3 b$ and $3 c$ ). Finally, acceleration seems to reduce the apparent duration of stimuli which follow the moving shape (Experiment 4).

These results are surprising and provide an important constraint on models of time perception. They pose two core questions. First, how are we to explain the differences in the apparent duration of constant-speed, accelerating, and decelerating objects? And second, why do the subjective durations indicated by the judgment tasks differ from those indicated by the reproduction tasks? These questions will be considered in turn, beginning with the latter.

\section{The dissociation between judged duration and reproduced duration}

Many studies use temporal reproduction to assess perceived duration (e.g., S. W. Brown, 1995; Kanai et al., 2006; Macar, Grondin, \& Casini, 1994; Woodrow, 1930). It is usually implicitly assumed that reproduction will produce the same qualitative pattern of results as other tasks, such as magnitude estimation. This was not found in the current experiments.

A tentative explanation is that the three types of movement differentially affected the passage of subjective time after stimulus offset. The reproduction tasks involved matching the duration of a static, post-movement display to the remembered duration of a dynamic object. In Experiment 4, the post-movement display appeared shorter after watching an accelerating shape than after a constant-speed or decelerating shape. That is, it will take longer for the post-movement display to "feel as long" after watching the accelerating shape as after the constant-speed or decelerating stimuli. We can therefore reconcile the estimation and reproduction results as follows. After stimulus offset, the encoded duration of the moving shapes follows the order constant speed > decelerating > accelerating, and participants' category judgments or magnitude estimates reflect this ordering. However, the movement also affects the apparent duration of the post-stimulus display such that it takes longer to reach a given subjective duration after viewing an accelerating shape. Thus, the temporal reproductions of the accelerating and decelerating shapes are very similar: the accelerating shape "seemed shorter" but it also takes longer for the post-stimulus display to match this encoded duration. This argument is speculative, but it is consistent with the pattern found in Experiments $3 a, 3 b, 3 c$ and 4 .

The idea that apparent duration depends upon the preceding stimulus is consistent with the general finding that perceptual (and non-perceptual) judgments are shaped by the previously judged 
item (e.g., DeCarlo \& Cross, 1990; Matthews \& Stewart, 2009a; Matthews \& Stewart, 2009b; Ward \& Lockhead, 1970), and with evidence that is more specific to time perception. For example, G.D.A. Brown et al. (2005) found that judgments of durations assimilated towards the stimulus presented on the previous trial. The precise mechanism underlying the effects of changes in stimulus speed on the perceived duration of subsequent items is a topic for future investigation. Broadly, we can distinguish two alternatives.

The first is that the effects result from the perceptual similarity between the successive stimuli. Novel stimuli seem to last longer than repetitions (Noguchi \& Kakigi, 2006; Pariyadath \& Eagleman, 2007, 2008; Tse et al., 2004), and it may be that the static displays which followed the moving shapes in Experiments $3 b, 3 c$, and 4 were more perceptually different from the constant speed and decelerating stimuli than they were from the accelerating stimuli, explaining the shorter apparent duration in the latter case. This suggestion is post-hoc, but it carries the prediction that the effect of stimulus motion on the apparent duration of subsequent stimuli will depend on the properties of those stimuli - their similarity to the moving shape.

The second possibility is that stimulus motion exerts a more general change on the passage of subjective time, such that the consequences will be the same irrespective of the properties of the subsequent stimulus. For example, changes in stimulus speed may affect the rate of an "internal pacemaker". Wearden and colleagues have found that playing a rapid train of clicks before stimulus presentation increases its perceived duration, and this effect increases with increasing stimulus length consistent with "speeding up" an internal clock (Penton-Voak, Edwards, Percival, \& Wearden, 1996; Wearden, Edwards, Fakhri, \& Percival, 1998; Wearden et al., 2007). The naive expectation might be that an accelerating stimulus will produce a faster subsequent pacemaker rate (i.e., the opposite of what was found here), but the situation is likely to be more complicated, depending on the characteristic frequency of the pacemaker, the frequencies of the moving stimuli, and the coupling between the two (see e.g., Treisman et al., 1990).

As a subsidiary exploration of this idea, I examined the delay between stimulus offset and the point where participants began reproduction in Experiment $3 \mathrm{~b}$. This latency may be taken as some indication of the rate of passage of subjective time, with longer latencies indicating slower rates. The results are plotted in Figure 5 (data treatment was as for the main analysis reported above, except that trials where the latency was longer than $2.5 \mathrm{~s}$ were excluded). A repeated measures ANOVA indicated no effect of stimulus duration and no interaction between duration and movement $[F(2.35,56.27)=1.92, p=.149$ and $F(6,144)=1.65, p=.139$ respectively $]$. However, the time that people waited before beginning the reproduction did depend on the nature of the stimulus movement, $F(1.51,36.12)=5.31, p=.016, \eta_{p}^{2}=.18$; people seem to wait longer in the accelerating 
condition than in the constant speed and decelerating conditions (Bonferroni-corrected $p s=.032$ and .065 ) which were very similar (corrected $p=1.00$ ). These results are therefore congruent with the framework outlined above, in which the passage of subjective time (pacemaker rate) is slower after viewing an accelerating shape - although of course other explanations of this pattern are also possible.

The core message is that temporal reproduction and temporal judgment need not produce identical results because the to-be-reproduced stimulus can influence the apparent duration of the subsequent interval during which reproduction takes place. The details of how this occurs comprise an important direction for future work. Additional problems with the use of temporal reproduction have recently been highlighted by Droit-Volet (2010).

\section{The effects of changes in speed on judgements of duration}

The primary topic of the current paper is the effect of changes in stimulus speed on apparent duration. Experiments 1, 2, and 3a found that stimuli moving with constant speed are judged to last longer than those which decelerate, which in turn seem to last longer than those which accelerate. This pattern is surprising - although Grassi and Darwin (2006) have reported a potentially related finding with auditory stimuli. In what follows I discuss how the current results relate to three broad accounts of time perception, focussing on general principles rather than specific theoretical models.

Memory, mental content, and the segmentation of experience. It has been argued that perceived duration depends on the "mental content" or complexity of a stimulus - the storage space needed to hold the event in memory (Ornstein, 1969). It is difficult to explain the current results in terms of stimulus complexity. The stimuli in all conditions moved the same distance over the same objective time. If anything, the accelerating and decelerating conditions represent greater complexity because they entail changes in stimulus speed as well as changes in stimulus position, yet it was the constant speed condition which appears to last longest.

Relatedly, several authors have suggested that perceived time depends on the number of changes during the interval, with more changes producing longer apparent durations (Block \& Reed, 1978; Poynter, 1989). For moving stimuli, it has been argued that moving stimuli seem to last longer than static ones because they entail more spatial change, and fast-moving stimuli seem to last longer than slow-moving ones for the same reason (e.g., Brown, 1995). It is unclear how this extends to the case where stimulus speed also changes. If apparent duration is solely dependent on changes in position then constant-speed, accelerating, and decelerating shapes should appear equal. To the extent that variations in speed represent an additional change, the accelerating and decelerating 
conditions should seem longer than the constant speed case. Neither pattern was found here. Finally, it might be possible to construct a change-based model in which the observer monitors the changes in spatial location on a moment-by-moment basis and integrates them over time; this leads to conclusions indistinguishable from those of the accumulator-based models discussed in the next section.

Complexity- and change-based accounts of perceived duration do not offer a straightforward explanation of the current data. More generally, it has been argued that such accounts are bettersuited to retrospective judgments - those in which the observer does not know in advance that a duration estimate will be required - where responses are based on remembered duration (Block \& Zakay, 1997; Grondin, 2001).

Counting, clocks, and accumulation. Many models assume that time perception is based on some kind of counting or accumulation process (e.g., Creelman, 1963; Gibbon et al., 1984; Killeen \& Weiss, 1987; Treisman, 1963; Treisman et al., 1990). There is usually assumed to be an internal pacemaker or oscillator which emits counts or pulses; stimulus timing involves counting the number of pulses which occur during the to-be-timed interval. There are numerous instantiations of this idea but for now it sufficient to focus on the common principle that timing involves accumulation, and it will be convenient to think of this accumulation as a time-continuous process. Manipulations which alter perceived duration are typically argued to affect the rate of accumulation (Matthews, Stewart, \& Wearden, in press; Wearden et al., 1998; Wearden et al., 2007), but they might also influence the latency with which timing is begun or ended (Matthews, in press).

How might an accumulator model accommodate the current results? An obvious approach is to assume that the rate of the accumulation depends upon the stimulus speed (Kaneko \& Murakami, 2009). Figure 6a schematically illustrates the changes in speed that took place over the course of stimulus presentation in the current experiments. The question then is: how do we relate these speeds to the accumulation rate in such a way that the total number of counts is in the order constant speed $>$ decelerating $>$ accelerating?

Consider first the fact that the constant speed case has the longest perceived duration. This rules out a linear relation between accumulation rate and speed: even if the rate at the beginning of the stimulus was weighted more heavily than the end (or vice-versa), the constant-speed case will be intermediate between the other two. A straightforward solution is to assume a non-linear coupling between stimulus speed and accumulation rate. Figure $6 \mathrm{~b}$ shows the results when accumulation rate is a logarithmic function of speed; other non-linear functions would serve as well, but there is some indication that a logarithmic function is a sensible choice. (In a recent study, Kaneko and Murakami (2009) examined the perceived duration of Gabor patches with a moving 
carrier and reported a logarithmic relationship between speed and subjective time.) In the figure, the relationship between accumulation rate and stimulus speed was modelled by $r=100+$ $100 * \ln (1+s)$, where $r$ is the rate of accumulation, $s$ is speed, and the extra constants were arbitrarily chosen to ensure that the accumulation always has a reasonably high non-zero rate and to avoid taking the logarithm of zero for stationary stimuli. The perceived duration is obtained by integrating the curves shown in Figure $6 \mathrm{~b}$. As required, the constant-speed stimulus has the longest subjective duration.

Under this scheme, the accelerating and decelerating stimuli will always have the same apparent duration because they are symmetric in time (the latter is equivalent to the former played backwards). The observed ordering decelerating > accelerating can be obtained if we assume that the counts from the early part of the stimulus are weighted more heavily than those from the end for example, because the observer is more attentive at the start of the stimulus. An attentional "gate" between pacemaker and accumulator which contracts over the course of stimulus presentation provides a possible mechanism for this (see below). Figure $6 \mathrm{c}$ shows the results of applying an exponentially-decreasing weighting function to the accumulation rates from Figure $6 \mathrm{~b}$. Specifically, the weighting function was $w=\exp (-0.1 t)$ where $t$ is the time since stimulus onset. Again, the functional form is arbitrary: other decreasing functions can produce the same effect. Integrating the curves in Figure $6 \mathrm{c}$ gives the required result: constant speed produces the longest subjective duration, followed by deceleration, with the accelerating shape having the shortest apparent duration. A different choice of parameters will produce different results, but the argument shows that the surprising pattern of results from the current experiments can be obtained by two reasonable assumptions - a non-linear relationship between stimulus speed and accumulation rate, and a differential weighting of information accumulated during early and late portions of the stimulus. Perhaps more importantly, any attempt to explain the current data in terms of an instantaneous coupling between accumulation rate and stimulus speed must incorporate both of these assumptions. A monotonic change in the weighting of pacemaker output over the course of the stimulus cannot by itself make the constant speed condition longest because the speed of the constant stimulus is always below that of one of the other two stimulus types (except at the middle of the presentation, when all three stimuli have equal speed). Similarly, an arbitrary relationship between pacemaker rate and stimulus speed cannot separate the accelerating and decelerating conditions because they are symmetric in time.

I have suggested that the results arise from a non-linear positive relationship between stimulus speed and accumulation rate, and a diminishing weighting of the pacemaker output over the course of the stimulus presentation. However, exactly the same pattern of results can be 
obtained by reversing these assumptions - that is, by positing that accumulation rate is inversely related to stimulus speed, with a weighting function that emphasizes the most recent portions of the stimulus. Greater weighting of more recent experience is plausible and might arise if, for example, there is passive leakage of accumulated counts (cf. Wackermann \& Ehm, 2006). The idea that the rate of accumulation is greater for slower-moving stimuli is perhaps counter-intuitive, and runs against the typical assertion that faster stimuli seem to last longer (Beckmann \& Young, 2009; S. W. Brown, 1995; Kaneko \& Murakami, 2009). However, there is some evidence that the relationship between speed and perceived duration is not uniformly positive (Predebon, 2002). Moreover, the fact that accelerating shapes (which end with rapid motion) make the subsequent static display appear shorter (Experiment 4) is consistent with a negative relationship between speed and pacemaker rate - although, as noted above, there are other explanations for this result. In order to clarify the relationship between pacemaker rate and stimulus speed, it would be helpful to compare the subjective durations of stimuli moving with different mean speeds over a range of durations for all three of the movement conditions studied here - constant speed, accelerating, and decelerating.

It has been assumed here that the coupling between stimulus speed and accumulation rate is instantaneous. More subtle versions are also possible, in which there is a lag between changes in stimulus speed and changes in accumulation rate. In addition, the foregoing assumes that the observer is able to estimate stimulus speed independently of the accumulation rate - for example, with relatively peripheral representations of speed feeding in to a more central pacemaker. However, it is also possible that the pacemaker is used when computing stimulus speed, with the observer determining how much the spatial position has changed between pulses and adjusting the pulse-rate contingent upon the computed velocity. The current data do not allow us to test these more complex formulations.

Attention and temporal processing. A related approach to time perception emphasizes the importance of attention. It is often argued that observers may attend to either the temporal or nontemporal aspects of a stimulus. Such accounts posit a limited attentional capacity which is shared between a timer (responsible for time processing) and a stimulus-processor (responsible for processing other stimulus features)(e.g., Thomas \& Weaver, 1975). The temporal processor accumulates "subjective time units" or pulses from a pacemaker (Zakay, 1989), and subjective duration is related to the attention given to time: greater temporal processing entails greater accrual of subjective time units and, in turn, longer apparent duration. Conversely, directing attention away from time - for example, by requiring judgments about some non-temporal aspect of the stimulus shortens apparent duration (Casini \& Macar, 1997; Macar et al., 1994). 
This class of model has much in common with those of the previous section. Indeed, Zakay and Block (1997) have modified the internal-clock model of Gibbon et al. (1984) to include an attentional gate which controls the flow of pulses from the pacemaker to the accumulator (see Lejeune, 1998, for a discussion). However, the attentional models incorporate an additional mechanism by which stimulus features or task demands may influence time perception: perceived duration may change because of a shift in the attention paid to non-temporal properties of the stimulus, rather than because of a change in the rate of the pacemaker.

Attentional accounts provide an alternative explanation for the finding that constant-speed stimuli seemed to have longer duration than accelerating or decelerating shapes. Suppose that attention can either be directed towards timing the moving objects or processing their other properties - including the pattern of their motion. For an object moving at constant speed, the motion presumably becomes predictable and uninteresting relatively quickly, freeing attentional resources to process the duration of the stimulus. When the stimulus speed continuously changes during viewing, more attention is devoted to processing its non-durational features over the whole course of presentation, entailing a shorter subjective duration. Additional assumptions would then be needed to accommodate the difference between accelerating and decelerating stimuli.

\section{Conclusion}

Changes in stimulus speed affect judged and reproduced duration - although not in the same way. The difference between the category rating/magnitude estimation tasks and the reproduction results is likely due to a change in the subjective passage of time during the reproduction interval; the basis for this will be an important direction for the future. It is too early to offer a definitive explanation of the effects of speed changes on judged duration. However, the results are not obviously accommodated by memory storage-based accounts of time perception, and if they are to be explained by pacemaker-accumulator models then a number of assumptions must be made about the relationship between stimulus dynamics and the rate of accumulation. The idea that changes in stimulus speed capture attention and prevent temporal processing provides an alternative perspective on the data, but additional assumptions will be needed in order to capture the full pattern of results.

The current work has focussed on simple comparisons between accelerating, decelerating, and constant-speed stimuli. A full understanding of how stimulus speed affects time perception will require an examination of additional parameters, including the starting, terminal, and mean speeds; the nature of the motion (e.g., linear vs. non-linear); the rate of acceleration/deceleration; and more complex changes in object speed over the course of stimulus presentation. 


\section{References}

Aubry, F., Guillaume, N., Mogicato, G., Bergeret, L., \& Celsis, P. (2008). Stimulus complexity and prospective timing: Clues for a parallel process model of time perception. Acta Psychologica, 128(1), 63-74.

Beckmann, J. S., \& Young, M. E. (2009). Stimulus dynamics and temporal discrimination: Implications for pacemakers. Journal of Experimental Psychology: Animal Behavior Processes, 35(4), 525-537.

Block, R. A., \& Reed, M. A. (1978). Remembered duration: Evidence for a contextual-change hypothesis. Journal of Experimental Psychology: Human Learning and Memory, 4(6), 656-665.

Block, R. A., \& Zakay, D. (1997). Prospective and retrospective duration judgments: A meta-analytic review. Psychonomic Bulletin \& Review, 4(2), 184-197.

Brown, G. D. A., McCormack, T., Smith, M., \& Stewart, N. (2005). Identification and bisection of temporal durations and tone frequencies: Common models for temporal and nontemporal stimuli. Journal of Experimental Psychology: Human Perception and Performance, 31(5), 919-938.

Brown, S. W. (1995). Time, change, and motion: The effects of stimulus movement on temporal perception. Perception \& Psychophysics, 57(1), 105-116.

Casini, L., \& Macar, F. (1997). Effects of attention manipulation on judgments of duration and of intensity in the visual modality. Memory \& Cognition, 25(6), 812-818.

Creelman, C. D. (1963). Human discrimination of auditory durations. Journal of the Acoustical Society of America, 34(5), 582-593.

DeCarlo, L. T., \& Cross, D. V. (1990). Sequential effects in magnitude scaling: Models and theory. Journal of Experimental Psychology: General, 119(4), 375-396.

Droit-Volet, S. (2010). Stop using time reproduction tasks in a comparative perspective without further analyses of the role of the motor response: The example of children. European Journal of Cognitive Psychology, 22(1), 130-148.

Erdfelder, E., Faul, F., \& Buchner, A. (1996). GPOWER: A general power analysis program. Behavior Research Methods, Instruments, \& Computers, 28, 1-11.

Gibbon, J., Church, R. M., \& Meck, W. H. (1984). Scalar timing in memory. In J. Gibbon \& L. Allan (Eds.), Annals of the New York Academy of Sciences, Volume 423: Timing and time perception. New York: New York Academy of Sciences.

Goldstone, S., \& Goldfarb, J. L. (1964). Auditory and visual time judgment. Journal of General Psychology, 70, 369-387.

Gomez, L. M., \& Robertston, L. C. (1979). The filled-duration illusion: The function of temporal and nontemporal set. Perception \& Psychophysics, 25(5), 432-438. 
Grassi, M., \& Darwin, C. J. (2006). The subjective duration of ramped and damped sounds. Perception \& Psychophysics, 68(8), 1382-1392.

Greenwald, A. J., Gonzalez, R., Harris, R. J., \& Guthrie, D. (1996). Effect sizes and $p$ values: What shouldbe reported and what should be replicated? Psychophysiology, 33, 175-183.

Grondin, S. (2001). From physical time to the first and second moments of psychological time. Psychological Bulletin, 127(1), 22-44.

Kanai, R., Paffen, C. L. E., Hogendoorn, H., \& Verstraten, F. A. J. (2006). Time dilation in dynamic visual display. Journal of Vision, 6(12), 1421-1430.

Kaneko, S., \& Murakami, I. (2009). Perceived duration of visual motion increases with speed. Journal of Vision, 9(7), 1-12.

Killeen, P. R., \& Weiss, N. A. (1987). Optimal timing and the Weber fraction. Psychological Review, 94(4), 455468.

Leiser, D., Stern, E., \& Meyer, J. (1991). Mean velocity and total time estimation effects of order and proportions. Journal of Environmental Psychology, 11(4), 347-358.

Lejeune, H. (1998). Switching or gating? The attentional challenge in cognitive models of psychological time. Behavioural Processes, 44, 127-145.

Lhamon, W. T., \& Goldstone, S. (1975). Movement and the judged duration of visual targets. Bulletin of the Psychonomic Society, 5(1), 53-54.

Macar, F., Grondin, S., \& Casini, L. (1994). Controlled attention sharing influences time estimation. Memory \& Cognition, 22(6), 673-686.

Masson, M. E. J., \& Loftus, G. R. (2003). Using confidence intervals for graphically based data interpretation. Canadian Journal of Experimental Psychology, 57(3), 203-220.

Matthews, W. J. (in press). Can we use verbal estimation to dissect the internal clock? Differentiating the effects of pacemaker rate, switch latencies, and judgment processes. . Behavioural Processes.

Matthews, W. J., \& Stewart, N. (2009a). The effect of interstimulus interval on sequential effects in absolute identification. Quarterly Journal of Experimental Psychology, 62(10), 2014-2029.

Matthews, W. J., \& Stewart, N. (2009b). Psychophysics and the judgment of price: Judging complex objects on a non-physical dimension elicits sequential effects like those in perceptual tasks. Judgment and Decision Making, 4(1), 64-81.

Matthews, W. J., Stewart, N., \& Wearden, J. H. (in press). Stimulus intensity and the perception of duration. Journal of Experimental Psychology: Human Perception and Performance. .

Miller, J. (2009). What is the probability of replicating a statistically significant effect? Psychonomic Bulletin \& Review, 16(4), 617-640. 
Noguchi, Y., \& Kakigi, R. (2006). Time representations can be made from nontemporal information in the brain: An MEG study. Cerebral Cortex, 16, 1797-1808.

Ornstein, R. E. (1969). On the experience of time. Harmondsworth, Middlesex: Penguin.

Pariyadath, V., \& Eagleman, D. M. (2007). The effect of predictability on subjective duration. PLOS ONE, 2(11), e1264.

Pariyadath, V., \& Eagleman, D. M. (2008). Brief subjective durations contract with repetition. Journal of Vision, 8(16), 1-6.

Peirce, J. W. (2007). PsychoPy - Psychophysics software in Python. Journal of Neuroscience Methods, 162(12), 8-13.

Penton-Voak, I. S., Edwards, H., Percival, A., \& Wearden, J. H. (1996). Speeding up an internal clock in humans? Effects of click trains on subjective duration. Journal of Experimental Psychology: Animal Behavior Processes, 22(3), 307-320.

Poynter, W. D. (1983). Duration judgment and the segmentation of experience. Memory \& Cognition, 11(1), 77-82.

Poynter, W. D. (1989). Judging the duration of time intervals: A process of remembering segments of experience. In I. Levin \& D. Zakay (Eds.), Time and human cognition: A life-span perspective (pp. 305321). Amsterdam: Elsevier.

Predebon, J. (2002). Stimulus motion and retrospective time judgments. Acta Psychologica, 109, 213-225.

Rammsayer, T., \& Ulrich, R. (2001). Counting models of temporal discrimination. Psychonomic Bulletin \& Review, 8(2), 270-277.

Thomas, E. A. C., \& Weaver, W. B. (1975). Cognitive processing and time perception. Perception \& Psychophysics, 17(4), 363-367.

Treisman, M. (1963). Temporal discrimination and the indifference interval: Implications for a model of the "internal clock". Psychological Monographs, 77(13), 1-31.

Treisman, M., Faulkner, A., Naish, P. L. N., \& Brogan, D. (1990). The internal clock: evidence for a temporal oscillator underlying time perception with some estimates of its characteristic frequency. Perception, $19,705-743$.

Tse, P. U., Intriligator, J., Rivest, J., \& Cavanagh, P. (2004). Attention and the subjective expansion of time. Perception \& Psychophysics, 66(7), 1171-1189.

Ulrich, R., Nitschke, J., \& Rammsayer, T. (2006a). Crossmodal temporal discrimination: Assessing the predictions of a general pacemaker-counter model. Perception \& Psychophysics, 68(7), 1140-1152.

Ulrich, R., Nitschke, J., \& Rammsayer, T. (2006b). Perceived duration of expected and unexpected sitmuli. Psychological Research, 70, 77-87. 
Wackermann, J., \& Ehm, W. (2006). The dual klepsydra model of internal time represenation and time reproduction. Journal of Theoretical Biology, 239, 482-493.

Ward, L. M., \& Lockhead, G. R. (1970). Sequential effects and memory in category judgments. Journal of Experimental Psychology, 84(1), 27-34.

Wearden, J. H., Edwards, H., Fakhri, M., \& Percival, A. (1998). Why "Sounds are judged longer then lights": Application of a model of the internal clock in humans. Quarterly Journal of Experimental Psychology, 51B(2), 97-120.

Wearden, J. H., \& Ferrara, A. (1993). Subjective shortening in humans' memory for stimulus duration. Quarterly Journal of Experimental Psychology, 46B(2), 163-186.

Wearden, J. H., Norton, R., Martin, S., \& Montford-Bebb, O. (2007). Internal clock processes and the filledduration illusion. Journal of Experimental Psychology: Human Perception and Performance, 33(3), 716-729.

Woodrow, H. (1930). The reproduction of temporal intervals. Journal of Experimental Psychology, 8(6), 473499.

Zakay, D., \& Block, R. A. (1997). Temporal cognition. Current Directions in Psychological Science, 6(1), 12-16. 


\section{Figure captions}

Figure 1. Results of Experiment 1. Error bars show standard errors. Heterogeneity of the error terms meant that it was not possible to use a pooled error term, so the error bars were calculated separately for each data point. For within-subjects and mixed designs these bars provide no indication of the significance of differences between conditions (Masson \& Loftus, 2003).

Figure 2. Results of Experiment 2. The key results are shown in the left panel, which plots the mean magnitude estimates. The right panel shows the coefficients of variation. Error bars show standard errors calculated separately for each data point and therefore provide no indication of the significance of differences between conditions.

Figure 3. Results of Experiments 3a (left panel), 3b (centre panel), and 3c (right panel). Experiment $3 a$ required category judgments; Experiments $3 \mathrm{~b}$ and $3 \mathrm{c}$ required temporal reproduction. Error bars show plus/minus one SEM, calculated separately for each data point.

Figure 4. Results of Experiment 4. The left panel shows the results organized by duration. Error bars show plus/minus one SEM calculated separately for each data point. The right panel shows the effects of stimulus motion, collapsed across duration. Here it was possible to obtain a pooled errorterm; the error bars show plus/minus $95 \%$ confidence intervals calculated for a within-subjects design.

Figure 5. Mean latency to begin reproduction in Experiment 3b, collapsed across stimulus duration. Error bars show plus/minus one SEM calculated separately for each data point (violations of sphericity prevented the use of a pooled term) so the bars are rather large and provide no indication of the significance of differences between means.

Figure 6. Schematic illustration of changes in stimulus speed and hypothetical changes in accumulation rate. The left panel shows stimulus speed over the course of presentation for each condition. The centre panel shows the corresponding accumulation rates, assuming a logarithmic dependency on stimulus speed. The right panel shows the effects of assigning progressively less weight to the pacemaker output as the stimulus proceeds. 
Figure 1.

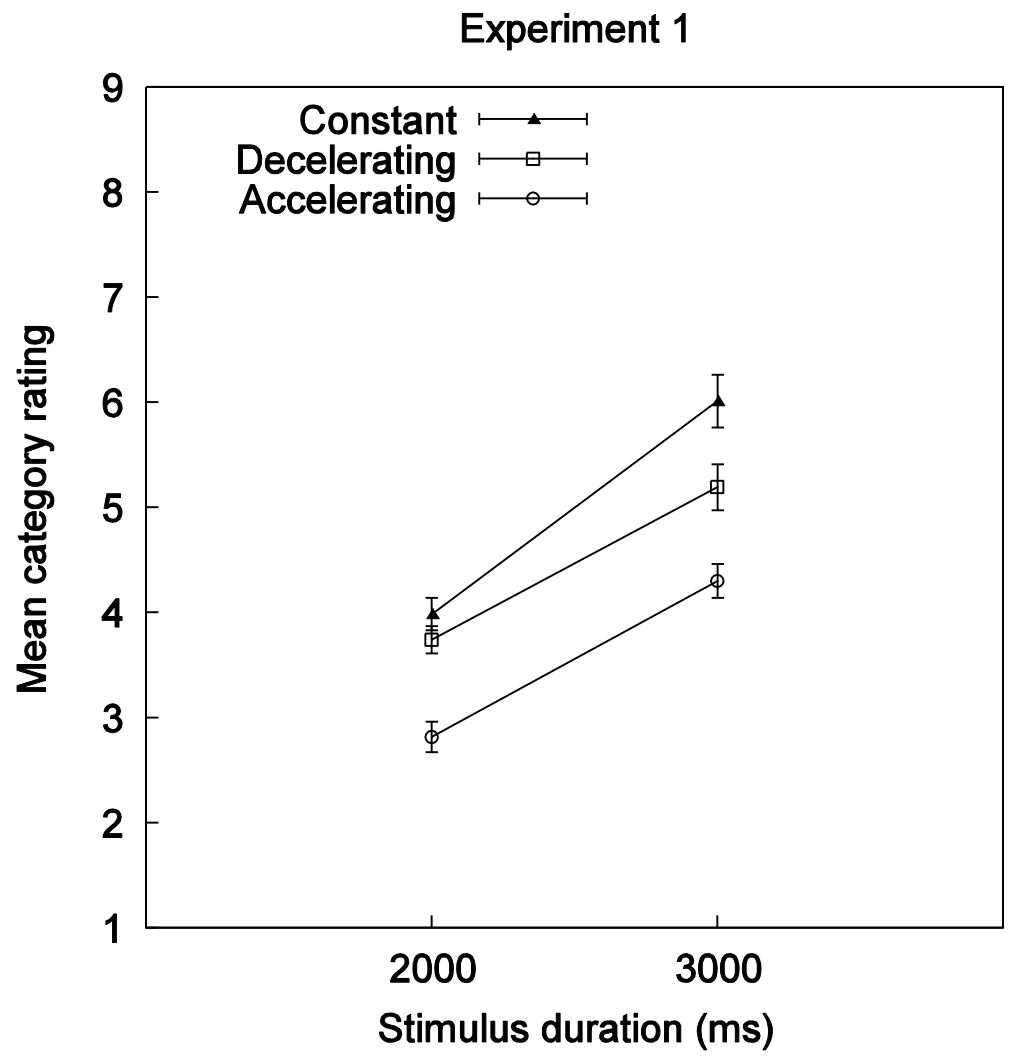


Figure 2.
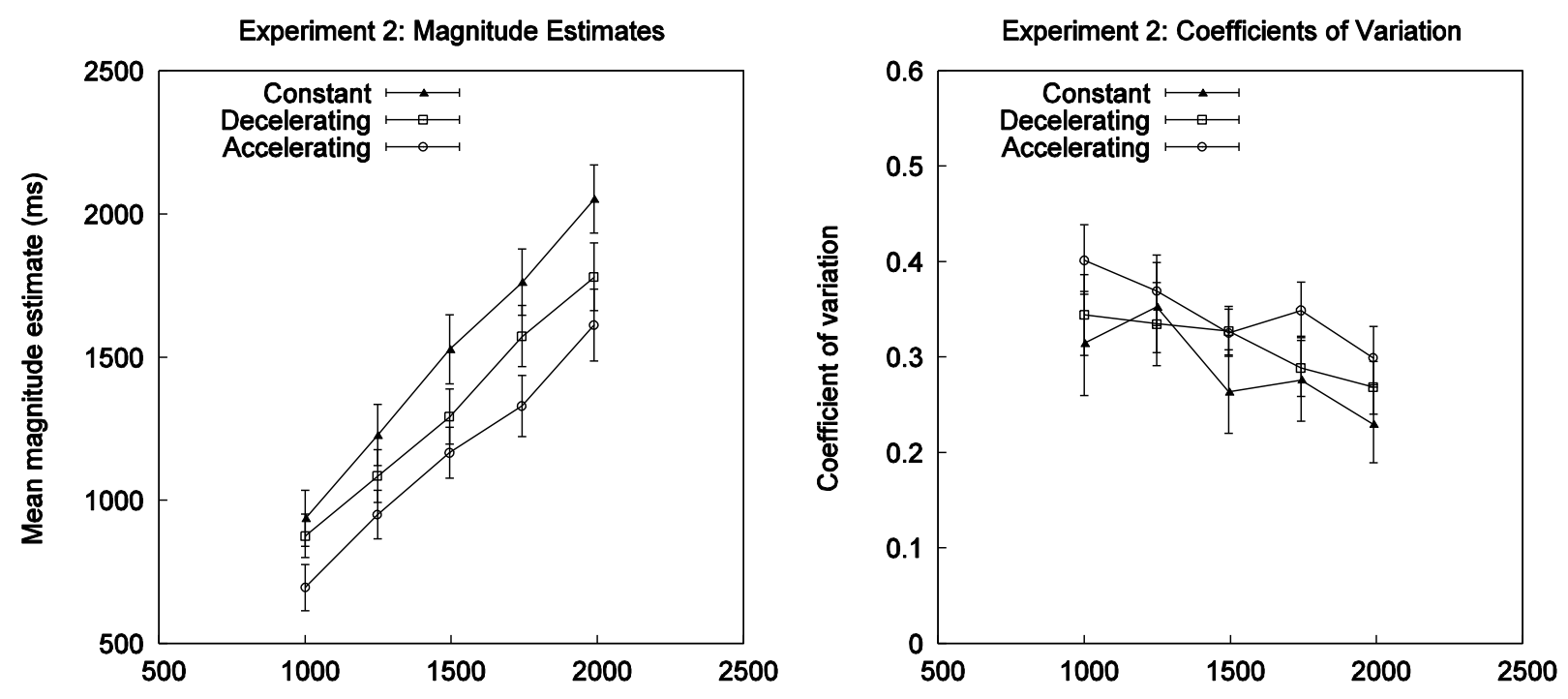
Figure 3.
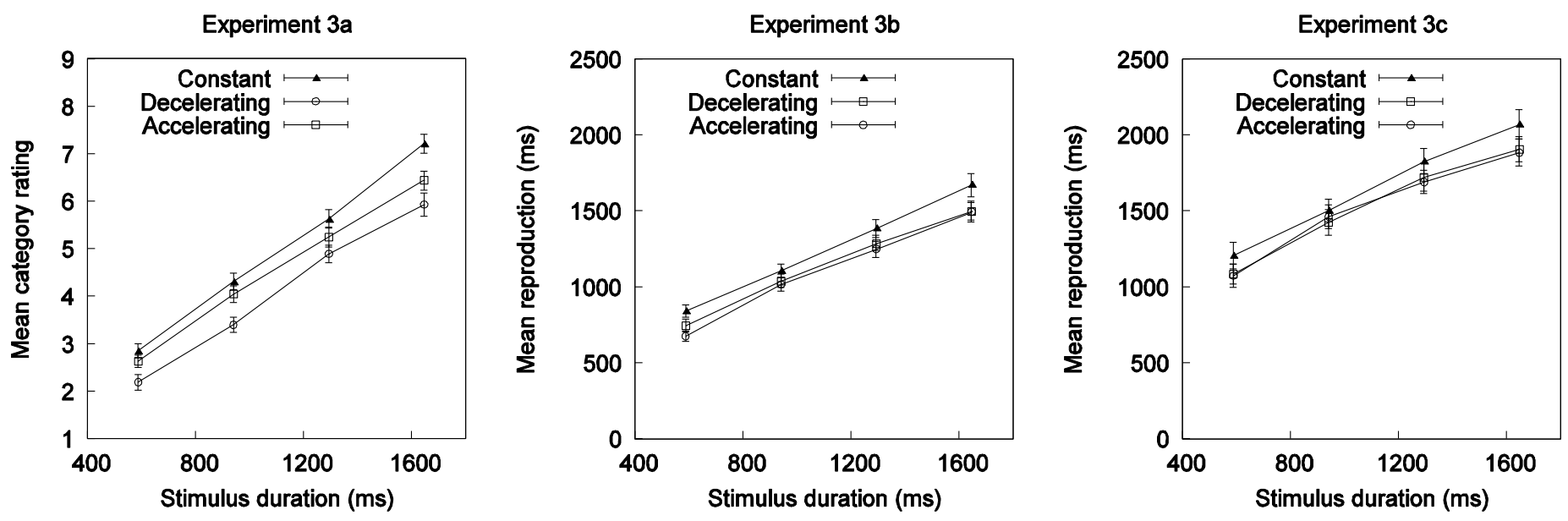
Figure 4.
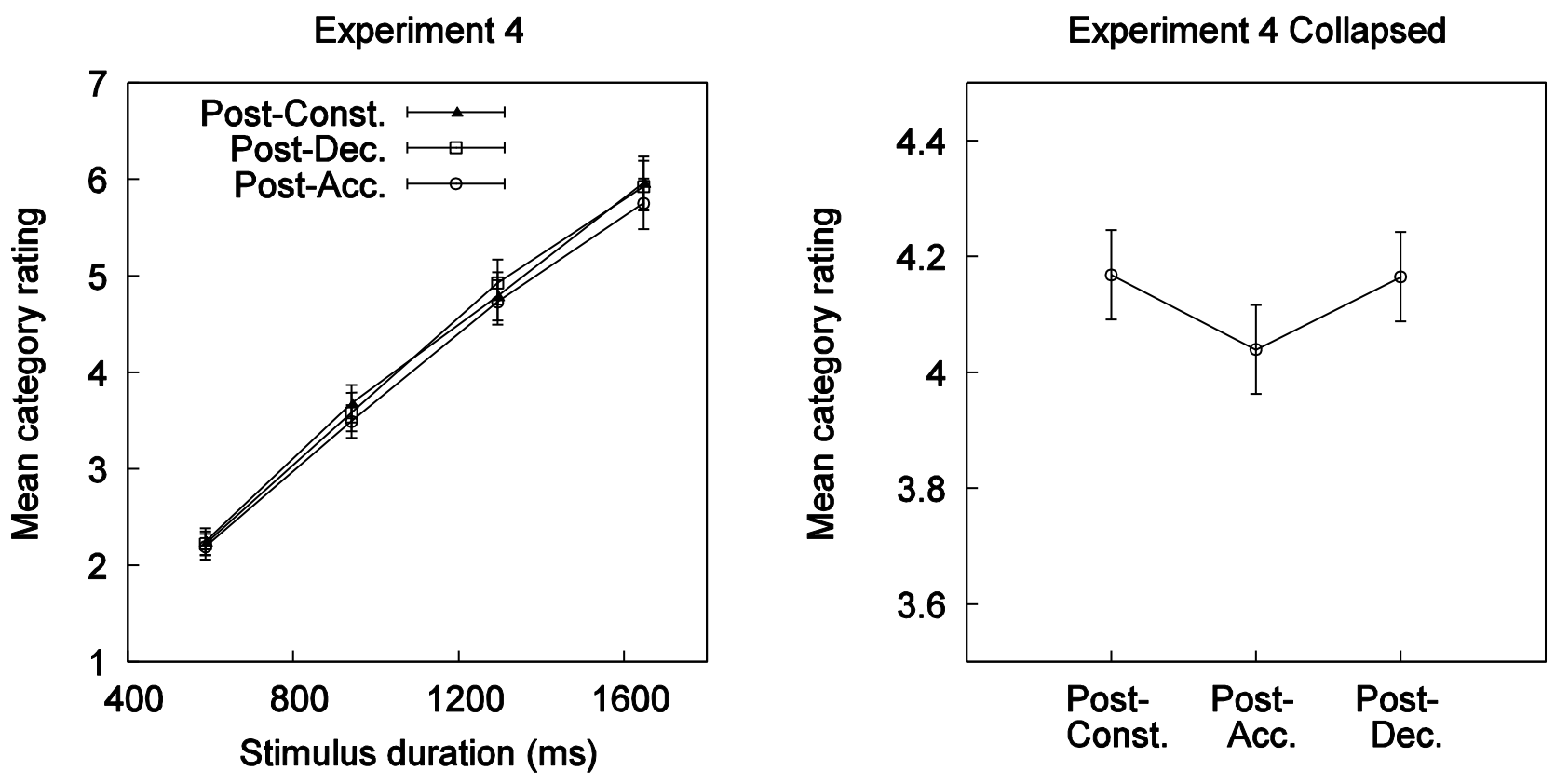
Figure 5.

Experiment 3b: Reproduction latencies

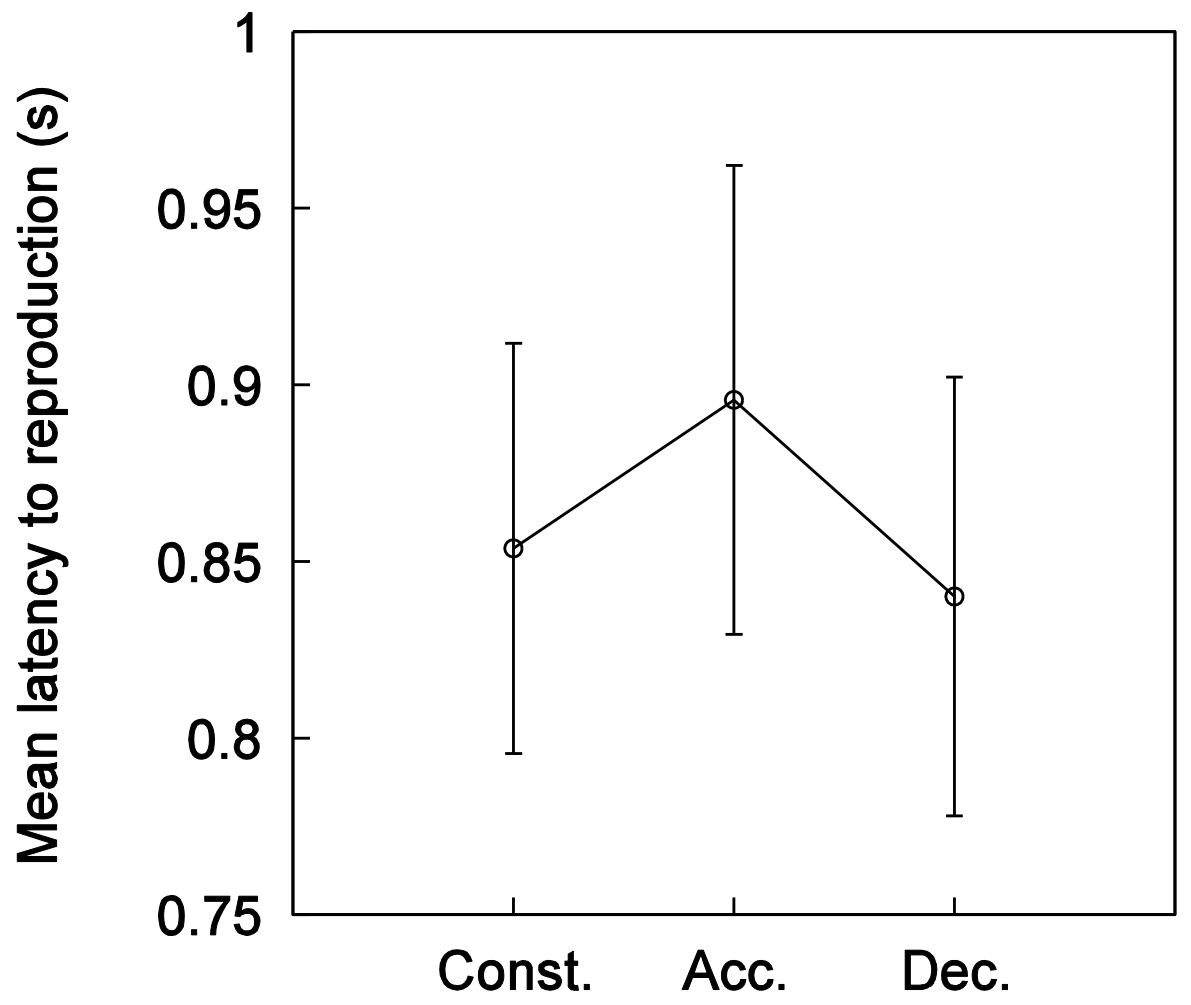


Figure 6.

(a) Speed over time

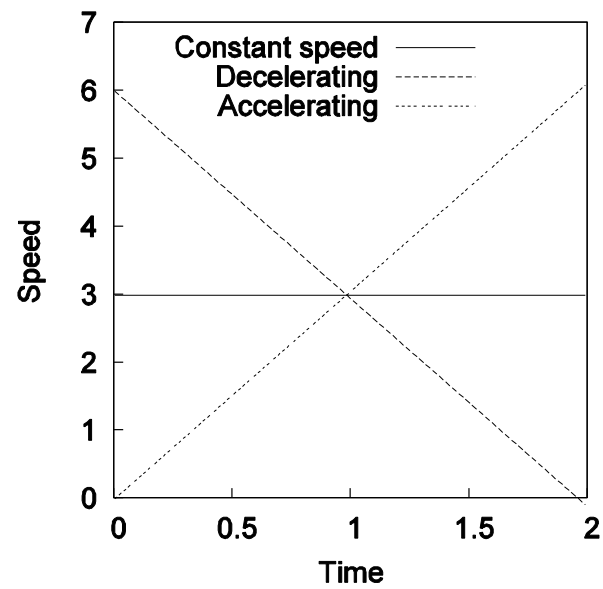

(b) Accumulation rate

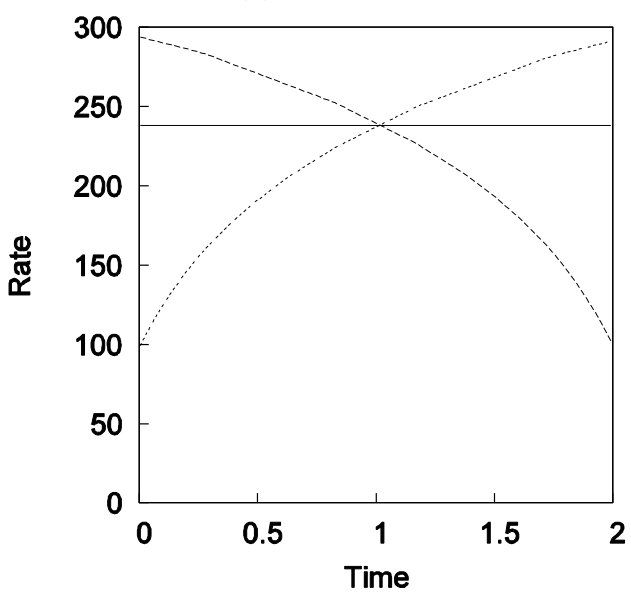

(c) Weighted accumulation rate

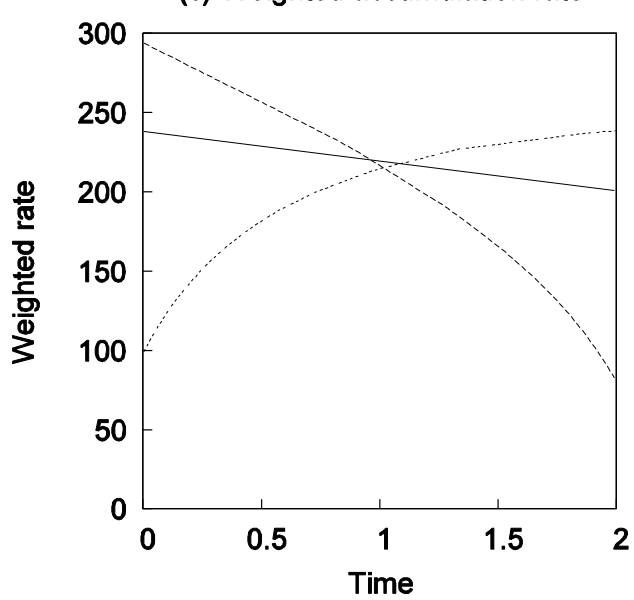

